\title{
Machine Learning and Big Data Analytics in IoT based Blood Bank Supply Chain Management System
}

\author{
J. Arul Valan ${ }^{1}$, Dr. E. Babu Raj² \\ ${ }^{1}$ Research Scholar, MS University, Tirunelveli \\ valanmspt@yahoo.co.in \\ ${ }^{2}$ Professor, Department of CSE, Marian Engineering College, Trivandrum \\ alanchybabu@gmail.com
}

\begin{abstract}
Blood is a perishable product with uncertainties in both supply and demand and blood stock management is therefore a judicious balance between shortage and wastage.Blood service operations are a key component of the healthcare system. Requirement of blood is increasing gradually due to accidents, surgeries etc. Blood transfusion play an important role in healthcare. The intention of Blood Bank Supply Chain is to demand estimate, inventory management and distribute adequate blood.Internet of Things (IoT) has rehabilitated the traditional e-healthcare system.Big data analytics refers to the process of collecting, organizing and analyzing large sets of data ("big data") to discover patterns and other useful information in a blood bank system. -Big DataAnalyticsandMachine Learning aretwo important areas ofdatascience. A key benefit of Machine Learning is the analysis and learning of massive amount so fun supervised data, making it a valuable tool for Big Data Analytics where raw data is largely unlabeled and un-categorized. With advance research in health sector, there is variety of perishable data available in health care especially in the Blood bank domain. This paper provides review and importance big data technologies and IoT paradigms used in health care sector.
\end{abstract}

Keywords - Internet of Things(IoT), Big Data, Machine Learning,Blood Bank, Supply Chain Management, Regional Blood Center (RBC), Hospital Blood Bank (HBB), Radio Frequency Identification (RFID).

\section{INTRODUCTION}

Internet of Things (IoT) has been emphasized as one of the new smart tool for the health care industry. IoT incorporates recent IT technologies that facilitate applications, devices, and objects to communicate among themselves through the connected networks (Tarouco et al., 2012). The IoT technologies primarily consist of data generation, collection, processing and distribution as well as big data management and service application. Initially, it has been used in the transportation monitoring and tracking, information pricing of products, and map navigation. However, the advance IT technologies such as wireless, cloud based network, smart phones, wearable devices, and high speed network connection have incorporated these IoT services into daily life activities. It has become a useful and necessity tool in the market and its popularity has been on the rise after the introduction of many new concepts such as Smart City, Smart Home, and Smart Health (Kang et al., 2015). According to the health context, the IoT has been widely used in various healthcare services (Fernandez and Pallis, 2014). In the IoT-based healthcare, there are interactions between things (applications) and humans (practitioners and patients) in order to obtain real-time data and support decision-making activities for care deliveries and other associated services. Moreover, the IoT would increase the utilization of resources in the healthcare environment, for example, managing limited resources for elderly care in the community more effectively (Feki et al., 2013; Li et al., 2011). The IoT-based healthcare system is able to connect to available resources in order to operate healthcare actions via the internet network. According to the benefits of the resource mobility, IoT concept could also be applied to manage other resources in the healthcare domain such as specimen, vaccines, and blood products. Blood is one of the most important resources in the healthcare system (Nagurney, Masoumi and Yu, 2012). Blood supply is also collected from human beings who have willingness to donate. Uncertainties in blood demand and supply are inevitable. Blood is perishable product, and its lifetime is within a short time period after collecting. This immense growth and evolution of data in health industry has given rise to lot of challenge like data collection, storage, transfer, processing and analysis [2]. . A key driver of this revolution is arguably "big data", which represents large volumes of machine-readable data [1]. Big data analysis is most important because the data 
is continuously changing based on interval of time to store big data most of companies are using cloud setup.Machine learning offers a solution to find patterns and association from thedata, which enables healthcare professionals to take precise decisions.Hence, if health care is combined efficiently with big data and machine learning, it results in effective storage, processing and analysis which can help to improve decision making process and finding better treatment solutions for present diseases [3].

\section{OVERVIEW OF IOT AND BIG DATA}

A. Internet of Things (IOT)

The term Internet of Things (IoT) generally refers to scenarios where network connectivity and computing capability extends to objects, sensors and everyday items not normally considered computers, allowing these devices to generate, exchange and consume data with minimal human intervention. Here we can divide the whole system into three basic components, ie., the Device,Gateway, and Cloud.

A device includes hardware and software that directly interact with the world. Devices connect to a network to communicate with each other, or to centralized applications. Devices might be directly or indirectly connected to the internet.

A gateway enables devices that are not directly connected to the Internet to reach cloud services. Although the term gateway has a specific function in networking, it is also used to describe a class of device that processes data on behalf of a group or cluster of devices. The data from each device is sent to cloudplatform, where it is processed and combined with data from other devices, and potentially with other business-transactional data.The internet of things has numerous applications in healthcare, from remote monitoring to smart sensors and medical device integration.

B. Big Data

Big data is the buzzword today. It is everywhere, especially in the healthcare industry. Traditionally, the huge amount of data generated by the healthcare industry was stored as hard copy. This data has the capability to support a wide range of healthcare and medical functions. The entirety of data that is related to patient health care and well-being makes up big data. Big Data has changed the way we manage, analyze and leverage data in any industry. One of the most promising areas where big data can be applied to make a change is healthcare. Big Data in healthcare is being used to predict epidemics, cure disease, improve quality of life and avoid preventable deaths. With the world's population increasing and everyone living longer, models of treatment delivery are rapidly changing, and many of the decisions behind those changes are being driven by data

\section{Overview of Machine Learning in Health Care}

Machine learning is one of the emerging area because it provides the ability to automatically attain deep insights, identify hidden patterns, and create predictive models from the preprocessed data, all without requiring explicit programming instructions and human interventions. Machine learning algorithms are classified as Supervised, Unsupervised and Reinforcement techniques. Deep learning also one of the machine learning algorithm and it is one of the significant algorithm used in health care sector. Successful application of machine learning in healthcare depends with intelligent algorithms and rich data sets. Self-driving car and websites that recommend items based on the purchasing decisions of other peopleare all examples of machine learning being used in the real world [12].

Machinelearningis one the valuable and necessary tool for the e-health care system that is used to combineandmakesenseofhealthcaredata. Perfect accurate diagnosis is achieved by Machine learning along with domain experts. The analysis of data is classified into descriptive, diagnostic, predictive, prescriptive and decisive categories. After the preprocessing of data, the analysis may be descriptive or diagnostic. But while we are analyzing present and historical data, the analysis will be predictive, prescriptive and decisive. Preprocessing plays a major role in the machine learning process. Preprocessed data is given as an input to the machine learning algorithm. Recently lots of innovative research going on in the medical field along with big data and machine learning. One phase of machine learning consists of data preprocessing plus learning. Efficient classification and prediction can be attained by proper machine learning algorithms. Models used in machine learning areClassification, Clustering and Regression [12].

\section{BLOOD BANK SUPPLY CHAIN MANAGEMENT (BBSCM)}

Practically, blood service operations involve blood collection, processing, inventory management, distribution, blood-banking management, and transfusion. 


\section{SUPPLY}

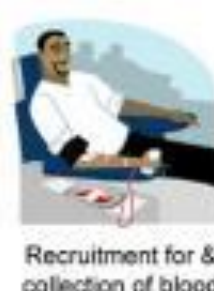

BLOOD SUPPLY-CHAIN
DEMAND

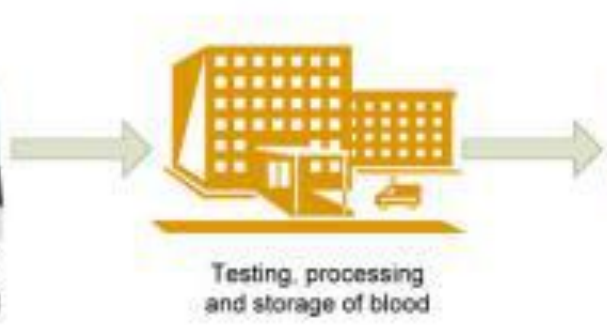

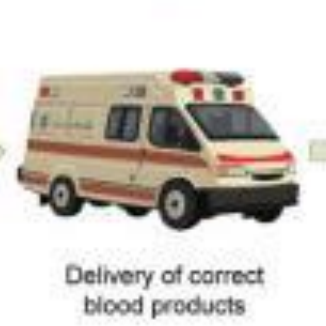

Delivery of carrect
blood products

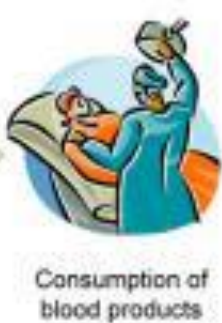

Fig.1: A simplified illustration of the blood supply-chain system

Blood Center collects whole blood from donors, processes it into blood products at a Regional Blood Center and distributes them to hospitals in the network in order to transfuse to the patient.Regional Blood Center $(\boldsymbol{R B C})$ has principal operations for blood collection, processing and testing, production, inventory management, and distribution to hospitals.RBC is responsible for managing this inventory and allocating available blood units to hospitals according to requisitions from their local blood banks.

Each Hospital Blood Bank (HBB) has to manage blood inventory and its operations within the hospital. Doctors put requests for the certain amount and groups of blood products for patients' treatments. When available blood products are assigned for any patient, these units will be crossmatched to verify the compatibility with each particular patient. The period between blood crossmatching and releasing any unused units is called "a period for reserving blood". The longer this period is, the higher the probability that blood will expire before its actual use. Finally, the crossmatched units will be transfused to the patient for treatment.

\section{IOT IN BLOOD BANK SUPPLY CHAIN}

According to the development in technologies, it would be beneficial to consider the possible advantages from using the IoT paradigm in the blood supply chain management.This paper provides conceptual guidelines in transforming blood service operations management to enhance blood utilization with the IoT-based applications paradigm.

\section{A.Tracking}

1) Inventory count and location tracking

Real-time inventory count would be applied to check the collaborative inventory in order to automated count the overall blood stock levels in the HBBs. Hence, the RBC can obtain these counted blood stock data for future blood collection and replenishment planning. Moreover, the location tracking of each blood unit would enhance blood transshipment among the HBBs in case there are any blood emergency demand requests made from the HBBs and the RBC cannot supply enough blood units to respond to those requests.

2) Safety and traceability

The blood traceability system is an online platform which is connected into the RFID-blood bag and barcode. It is able to integrate the sharing information and coordination among blood service organizations in the supply chain in order to mitigate blood transfusion risks to the patient, such as human error, incorrect blood products, and errors in medication administration (Dzik, 2007).

\section{B. Identification and Authentication}

1) Auto ID/bar code enabled transfusion administration

The identity check between each patient and particular blood product is the crucial task to avoid mistake in the transfusion. The RFID-blood bags and the traceability system can ensure that the correct blood type with the correct quantity is always delivered to the right patient when there is a request (Koshio and Akiyama, 2009).

\section{Automatic Data Collection}

\section{1)Blood inventory management}

Blood inventory is a major task in the supply chain. The main aim is to maximize blood utilization in such a way that blood shortage and outdated rates are at minimum levels. RFID and barcode are used to connect each blood bag to the blood inventory system can lead to the ability to manage blood information more efficiently. 2) Blood tracking and tracing

Real-time blood tracking and tracing system will help the RBC to reload its own inventory and allocate blood products to the hospital blood banks according to the specific demand from each hospital.

D. Sensing

1)Transportation monitoring

Temperature control during transportation is vital for maintaining blood quality. Using sensing technology and real-time temperature tracking in blood transportation can ensure the quality of blood products. 
2) Patient monitoring

It is necessary for the medical practitioners to be alerted if the patient suffers any complication symptoms after receiving blood. A wearable bio-signal device that binds the patient to the system can be used to monitor and transmit data automatically back to the medical staff for any prompt treatments during postoperative blood transfusions.

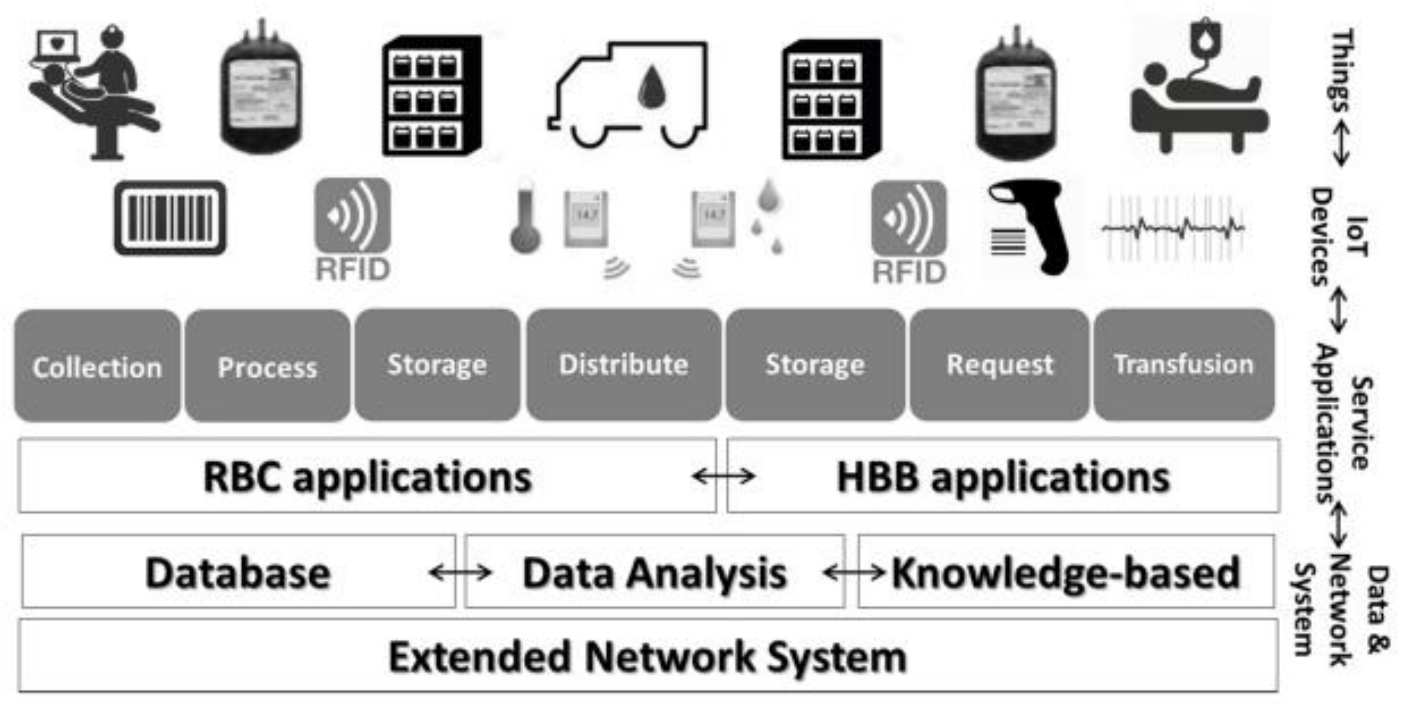

Fig.2.IoT-based framework for blood bank supply chain management

\section{BIG DATA ANALYTICS IN BLOOD SUPPLY CHAIN}

Big data is saving lives and it has been changing lives in many ways. Big data analytics is bringing a welcome shift in the healthcare sectors. Healthcare analytics cannot only help reduce the cost of healthcare facilities including treatments, medication, and diagnosis. Over the past decade, Electronic Health Records (EHR) have been widely adopted in hospitals and clinics worldwide. Important clinical knowledge and a deeper understanding of patient disease and symptoms patterns can be studied from such data. It will help to improve patient care and improve efficiency.

Using automatic data collection and transferring enables the blood management system to store a large amount of useful data in the blood service organizations. In developed countries, a blood-banking system which links between blood centers and hospitals can be performed automatically to communicate or make decisions on blood service operations. It is necessary to have information stored in the blood management system to use as a knowledge tool for supporting future decisionmakings in any levels such as blood allocation policy, blood inventory management, and blood cross-matching policy.Hence, if healthcare is combined efficiently with big data techniques it results in effective storage, processing and analysis which can help to improve decision making process and finding better treatment solutions for present diseases.

\section{Applications of Big Data and Machine Learning in Health Care}

E- healthcare industry is being transformed by the advancements in machine learning and Big data analytics. Machine learning and big data are now being used in healthcare to provide superior patient care and has resulted in improved predicted outcomes. Nowadays, machine learning supports personalized health care through improved diagnostics and predictive \& prescriptive healthcare analytics. Machine learning algorithms process huge structured and unstructured datasets (big data) and provide useful perceptions that allow effective health care services and it can make accurate decisions, significantly improve operating efficiencies, and reducing the unwanted cost.

Monitoring patient vitals: Sensors are being used in the patient beds to continuously monitor blood pressure, heartbeat and respiratory rate. Any deviations in pattern is immediately reported to doctors and healthcare adminis trators [7].

Healthcare Intelligence:Machine learning and Big Data are being used in healthcare Intelligence applications [7]. Fraud Prevention and Detection:Machine learning algorithms helps to avoid a wide range of human errors on the side of health care administrators in the form of dosage level, medicines, and other kinds of treatments. It will also be used to detect and prevent fraudulent claims of insurance from the insurance agencies [7].

Real-time Alerting: Wearable IoT enabled devices will collect patients' health data continuously and send this data to the cloud setup.This information will be accessed 
from the cloud databases on the state of health of the general public, which will allow doctors to compare this data along with existing patient record. For example, if patient's blood pressure increases, the system will immediately send an alert to the doctor who will then take care the immediate action [8].

Predictive Analytics in Healthcare: One of the key functionality of machine learning algorithm is Predictive Analytics. In health industry, it will save human lives.It will help doctors to make decisions within seconds and improve patients' treatment. This is particularly useful in case of patients with complex medical histories, suffering from multiple conditions [8].

Evidence-Based Medicine: It is one of the remarkable application of machine learning. Using evidence-based medicine, the doctor can compare symptoms of a patient to a larger patient database in order to come to an accurate diagnosis faster and more efficiently. Here big data role is assimilating information from different sources and normalizing the data in an accurate manner [9]

Analyzing Hospital Networks: With the help of the recent IoT enabled cloud based technologies we can easily tracking and monitoring the facility, availability of specialist doctors, nearby hospitals and locations of the hospitals [9].

\section{CHALLENGES}

Blood supply chain is a challenging system to manage. Its overall operations are complex which are associated with human, blood centers, hospitals, and patients. Presently, there are advanced medical technologies that support various aspects of e-health services in order to improve the health outcomes of patients as well as enhance performances of care delivers, including Telemedicine, E-health, and IoT. Despite these benefits, Llewellyn et al. (2014) addressed barriers to the adoption and implementation of the advanced medical technologies. These barriers involve with the lack of encouragement from the Department of Health, change issues in new operational procedures, skills of practitioners, threats perception, leadership, infrastructure, project management, lack of knowledge towards new technologies, and costs. In order to overcome these obstacles, the development of the IoT application in the blood operations system is required to guarantee that such technologies are trustworthiness for investment in the real context.

\section{CONCLUSION}

Nowadays, health care systems are rapidly adopting clinical data, which will rapidly enlarge the size of the health records that are accessible, electronically. A robust, sustainable blood system is a crucial component of every health care system. The availability of safe blood and blood products is a prerequisite for various health care services-including some surgeries, treatments for cancer and other acute and chronic medical conditions, trauma care, organ transplantation, and childbirths - that extend and improve life for millions of patients annuallyIoT-based healthcare applications paradigm consists of four main functions, tracking, identification and authentication, automatic data collection, and sensing. This study has incorporated the IoT paradigm to provide the guidelines to transform the blood supply chain management in order to improve blood service operations. Tracking is used to monitor blood inventory counting and storage location. Identification and authentication is the function aimed to ensure that the blood transfusion standard is followed. Automatic data collection is used as a mechanism to drive the information flows in blood logistics operations. The IoT-based framework of the blood supply chain management is proposed to conceptualize the interactions between blood products, donors, patients, practitioners, processes, and service operation applications through the network connection. The consolidation of the blood service organizations is a key success towards the implementation of IoT-based applications with big data analytics in the blood bank supply chain management.

\section{REFERENCES}

[1] Bui, N. and M. Zorzi (2011). "Health care applications: a solution based on the Internet of Things". In: International symposium on applied sciences in biomedical and communication technologies. Ed. by ACM. New York, p. 131.

[2] Dobbin, J., R. Wilding, and S. Cotton (2009). "True blood: challenges of the blood supply chain in England". In: Logistics \& transport focus 11.11, pp. 32-36.

[3] Doukas, C. and I. Maglogiannis (2012). "Bringing IoT and cloud computing towards pervasive healthcare". In: International conference on innovative mobile and internet services in ubiquitous computing (IMIS), pp. 922-926.

[4] Dzik, W. H. (2007). "New technology for transfusion safety". In: British journal of haematology 136.2, pp. 181-190.

[5] Feki, M. A., F. Kawsar, M. Boussard, and L. Trappeniers (2013). "The Internet of Things: the next technological revolution". In: Computer 46.2, pp. 24-25.

[6] Fernandez, F. and G. C. Pallis (2014). "Opportunities and challenges of the Internet of Things for healthcare: systems engineering perspective". In: International con-ference on 
wireless mobile communication and healthcare transforming healthcare through innovations in mobile and wireless technologies (MOBIHEALTH), pp. 263-266.

[7] Kang, Y., M. Han, K. Han, and J. B. Kim (2015). "A study on the Internet of Things (IoT) applications". In: International journal of software engineering and its applications 9.9, pp. 117-126.

[8] Koshio, A. and M. Akiyama (2009). "Blood transfusion and patient safety with IT - minimizing risk of transfusion with Point-of-Act-System". In: International conference of the Asia-Pacific Medical Informatics Association, pp. 46-53.

[9] Li, X., R. X. Lu, X. H. Liang, X. M. Shen, J. M. Chen, and X. D. Lin (2011). "Smart community: An Internet of Things application". In: IEEE Communication magazine 49.11, pp. 68-75.

[10] Nagurney, A., A. H. Masoumi, and M. Yu (2012). "Supply chain network operations management of a blood banking system with cost and risk minimization". In: Computational management science 9, pp. 205-231.

[11] Pierskalla, W. P. (2005). "Supply chain management of blood banks". In: Operations research and health care, pp. 103-145.

[12] Rytila., J. S. and K. M. Spens (2006). "Using simulation to increase efficiency in blood supply chains". In: Management research news 29.12, pp. 801-819.

[13] Schreier, G. (2010). Pervasive healthcare via the Internet of Medical Things. Graz, Austria: Austrian Institute of Technology GmbH.

[14] Tarouco, L. M. R., L. M. Bertholdo, L. Z. Granville, L. M. R. Arbiza, F. Carbone, M. Marotta, and J. J. C. de Santanna (2012). "Internet of Things in healthcare: interoperability and security is sues". In: conference on communications (ICC). Ed. by I. International. Ottawa, pp. 6121-6125.

[15] Wijai Boonyanusith, Phongchai Jittamai, 'Transforming Blood Supply Chain Management with Internet of Things Paradigm', Proceedings of the Hamburg International Conference of Logistics (HICL) -23 